\title{
Direct costs of three models for the screening of latent tuberculosis infection
}

\author{
P. Wrighton-Smith* and J-P. Zellweger ${ }^{\#}$
}

ABSTRACT: The aim of the present study was to compare the direct costs of three models for detection of latent tuberculosis infection (LTBI) in routine clinical practice in Switzerland.

Comparison of the overall costs of screening for LTBI, including medical and radiological examination, and preventive treatment associated with three screening models was carried out. Model 1 relies only on the tuberculin skin test (TST) according to the current national guidelines, model 2 relies on T-SPOT ${ }_{\ltimes}$.TB (Oxford Immunotec, Oxford, UK) only and model 3 relies on TST followed by confirmation of positive results by T-SPOT.TB. Costs were taken directly from the clinic's figures. Clinical assumptions were based on the 267 patients who were referred to the clinic over the study period.

Model 3 was found to be the most cost-effective. Using only the skin test (model 1) was the least cost-effective. If only one test for LTBI is to be used, then model 2 (using T-SPOT.TB only) is cheaper than using the TST (model 1).

Screening for latent tuberculosis infection by tuberculin skin test followed by confirmation with T-SPOT.TB is less costly than screening with tuberculin skin test alone, as it allows a reduction in the number of people who receive preventive treatment. In groups with a high proportion of negative tuberculin skin tests, screening with T-SPOT.TB test only may be the most cost-effective.

KEYWORDS: Contact tracing, interferon- $\gamma$ release assays, latent tuberculosis infection, screening, tuberculosis

A key component of tuberculosis (TB) control is the targeted identification and treatment of people carrying a latent TB infection (LTBI), who are at elevated risk of progression to active TB disease [1]. To date, the tuberculin skin test (TST) has been relied on to identify those infected who would benefit from treatment. The recent advent of a rapid technique for detecting antigen-specific T-cells direct from blood offers a new approach for detecting Mycobacterium tuberculosis infection $[2,3]$. The TSPOT®.TB (Oxford Immunotec, Oxford, UK) test is the regulatory-approved version of the ex vivo enzyme-linked immunospot (ELISPOT) assay for the detection of activated T-cells specific for early secreted antigenic target (ESAT)- 6 and culture filtrate protein (CFP)-10 [4]. These antigens are present in $M$. tuberculosis but absent from Bacillus Calmette-Guerin (BCG) and most environmental mycobacteria $[5,6]$. The test is a sensitive and specific marker of $M$. tuberculosis infection, gives uniformly negative results in healthy unexposed controls (irrespective of BCG vaccination status), and correlates with $M$. tuberculosis exposure among contacts [7-17]. Given the lack of a gold

For editorial comments see page 1. standard for LTBI, these results strongly suggest that this may be a more accurate test than the TST. Other new blood tests using different methodologies based on the principle of detecting interferon- $\gamma$ released in response to stimulation by ESAT- 6 and CFP-10 have also been shown to be able to effectively discriminate TB infection from prior BCG vaccination [18, 19].

As a result of this clinical evidence, there have been growing calls to implement new blood tests into routine clinical practice. However, to date, there has been no published evidence on the cost implications of doing so.

The purpose of the present study is not to examine the absolute cost-effectiveness of LTBI treatment and screening programmes per se, but rather to examine the particular question of whether the new blood test for detection of LTBI (T-SPOT.TB) is cost-effective relative to existing programmes based upon the TST for screening recent contacts of infectious active TB patients. The study was designed to be based on actual clinical and cost data, and to examine the direct costs of implementation of this new blood test to the healthcare provider in terms of their overall budget for latent TB screening and treatment.
AFFILIATIONS

*Oxford Immunotec, Oxford, UK \#University Medical Policlinic, Lausanne, Switzerland

CORRESPONDENCE

J-P. Zellweger University Medical Policlinic Rue du Bugnon 44 1011 Lausanne Switzerland Fax: 41213144740 E-mail: Jean-Pierre.Zellweger@ hospvd.ch and zellwegerjp@ swissonline.ch

Received:

January 152006 Accepted after revision: March 292006 


\section{MATERIALS AND METHODS Participants}

The data for the analysis came directly from the use of the TST side-by-side with T-SPOT.TB under routine programme conditions at the University Medical Policlinic Lausanne (UMPL, Lausanne, Switzerland). The present authors have been running a study to compare the performance of the two tests in a number of population groups including recent contacts of infectious TB patients. All contacts presenting to the UMPL between January 2004 and December 2005 were included in the present study. A total of 267 participants were included in the study; 144 were Swiss-born and the majority of the foreignborn were immigrants from high incidence countries. In total, 168 had known BCG vaccination histories, with 89.9\% (151/ 168) having received the BCG vaccine.

The TST was applied using the Mantoux method, using 2TU of RT23 PPD, according to the Swiss National Guidelines [20]. Results were read at $72 \mathrm{~h}$ and considered positive if induration was $\geqslant 10 \mathrm{~mm}$. All TSTs were placed and interpreted by experienced operators. For the T-SPOT.TB test, a $10 \mathrm{~mL}$ blood sample was taken and analysed in a local analytical laboratory (BBR-LTC Laboratories, Lausanne, Switzerland). The cut-off for the assay was $\geqslant 6$ spots, according to the manufacturer's instructions.

According to the Swiss National Guidelines, contacts with a TST $\geqslant 10 \mathrm{~mm}$ are offered a preventive treatment with isoniazid for 9 months. Contacts who refuse the preventive treatment are offered a clinical follow-up for 2 yrs.

The raw data from the results of both tests, used in the subsequent cost-effectiveness analysis, are shown in table 1. Of the 267 contacts, $193(72.3 \%)$ were positive by TST. Seventyfour $(27.7 \%)$ were positive by T-SPOT.TB. Only 33.7\% (65 out of 193) of the TST-positive individuals were also positive by the T-SPOT.TB test. There were nine individuals who were TSPOT.TB positive and TST negative.

\section{Models}

A simple decision-tree analysis was constructed representing different possible clinical pathways for the diagnosis and subsequent treatment of LTBI (fig. 1). Three scenarios were modelled as follows: model 1 captures current practice where the TST is used as the only tool to diagnose LTBI, model 2 calculates the costs of using T-SPOT.TB alone as the only tool to diagnose LTBI (i.e. a complete replacement for the TST), and model 3 calculates the costs of using the TST for the initial screening of patients, followed by a T-SPOT.TB test in all

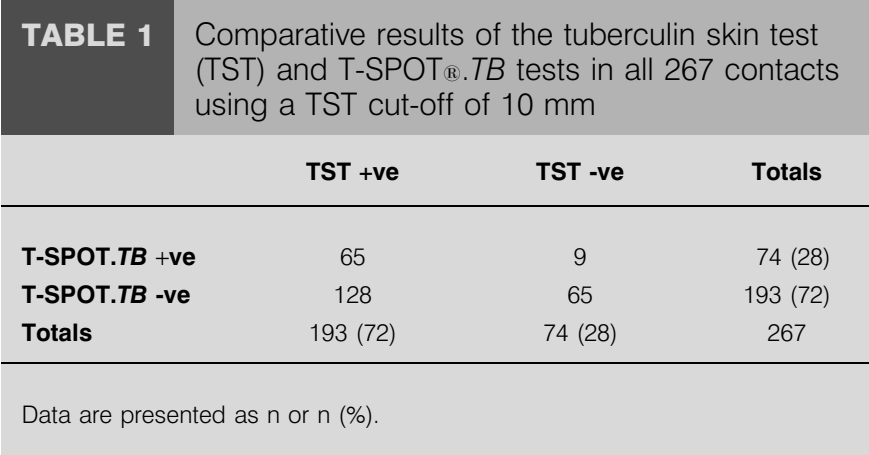

TST-positive individuals prior to treatment. The clinical and cost-effectiveness basis for model 3 has also been put forward by the UK National Institute for Clinical Excellence (NICE) in draft guidelines for the implementation of new blood tests for TB [21].

Each model was run for a hypothetical cohort of 1,000 patients, with the model inputs for the test results directly extrapolated from the actual results shown in table 1.

The models represent a direct-cost analysis from the point of view of the healthcare provider. No consideration was given to costs and quality-of-life losses on the part of the patient; in
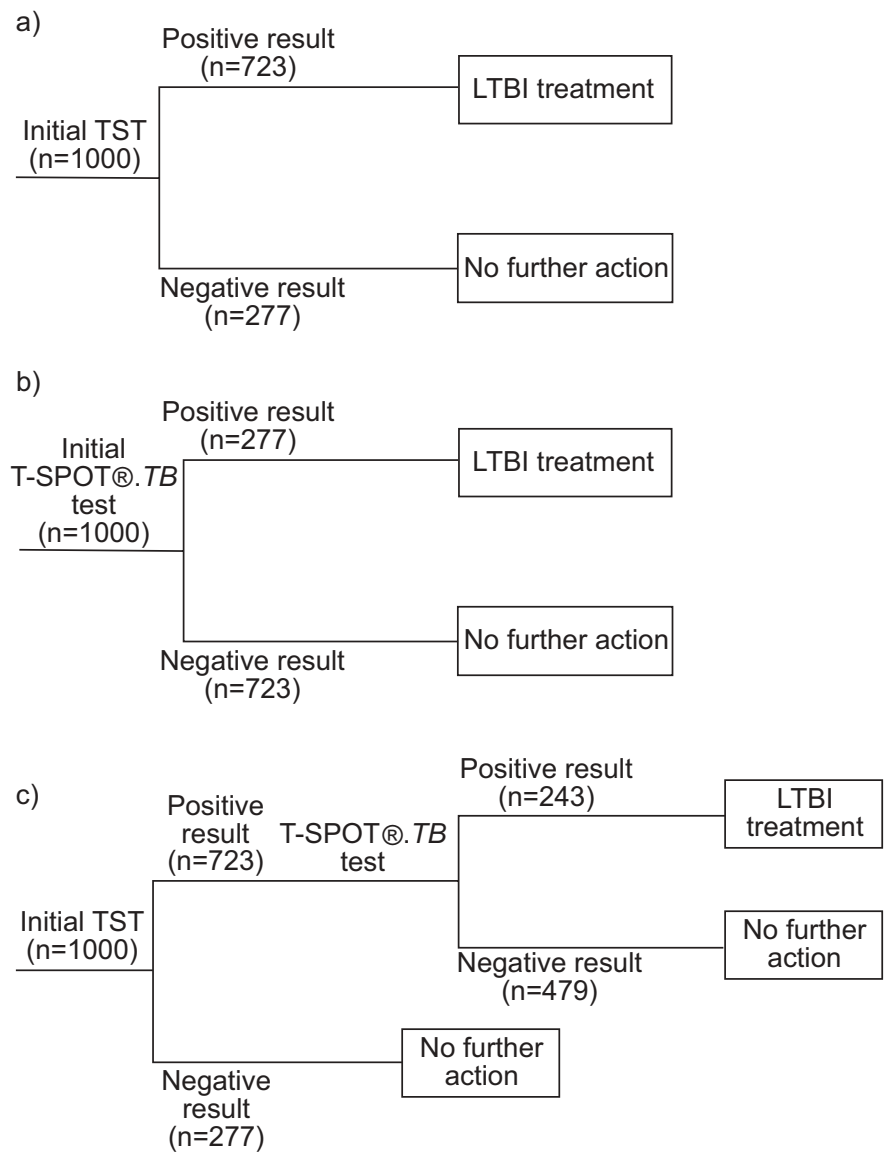

FIGURE 1. The three decision-tree clinical pathway models (models 1-3). a) Model 1: base case - costs of screening using the tuberculin skin test (TST) alone according to the Swiss guidelines [1]. b) Model 2: T-SPOT screening if T-SPOT.TB were to replace TST entirely as the only tool for detecting latent tuberculosis infection (LTBI). c) Model 3: T-SPOT.TB as confirmatory test costs of screening using TST as the first-line tool, followed by T-SPOT.TB in TST +ve individuals to rule out false positives. The overall cost results, with data and costs normalised to a cohort size of 1,000 subjects and using a skin test cut-off of $10 \mathrm{~mm}$ according to the Swiss guidelines [1] are as follows. a) Total cost of initial screening $=$ CHF35,000 (€22,575); total cost of treatment $=$ CHF1, 043,790

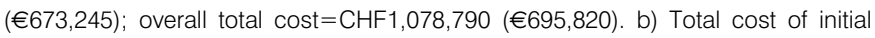
screening $=\mathrm{CHF200,000}(€ 129,575)$; total cost of treatment $=\mathrm{CHF} 400,210$ $(€ 258,135)$; overall total cost $=\mathrm{CHF} 600,790(€ 387,135)$. c) Total cost of initial screening $=\mathrm{CHF} 35,000$ (€22,575); total cost of confirmatory test CHF144,569 $(€ 93,247)$; total cost of treatment $=$ CHF351,536 $(€ 226,740)$; overall total cost $=$ CHF531,105 (€342,563). CHF: Swiss Francs. 
addition, no consideration was given to wider transmission of $\mathrm{TB}$ in the community. No time discounting was necessary, as all the costs in each clinical pathway were assumed to be incurred during a 1-yr period. The present authors also did not account for the costs of repeated TSTs, which are in practice often administered in dubious or potentially boosted cases.

Costs were taken directly from the published dispensary costs at the UMPL as shown in table 2, and expressed in both Swiss Francs $(\mathrm{CHF})$ and Euros (at a rate of $\mathrm{CHF} 1=€ 0.645)$. The cost of LTBI treatment includes an initial chest radiograph to rule out active TB prior to treatment, the cost of 9 months of isoniazid and the cost of clinician visits and liver function tests during the treatment period. Costs associated with bacteriological examination of sputum, if indicated, and side-effects from isoniazid treatment were ignored. All patients in whom preventative therapy was indicated were assumed to complete the full course of therapy. As no reimbursement amount has yet been established in Switzerland for the T-SPOT.TB test, a figure of CHF200 ( $€ 129)$ was taken as an initial sum taken to estimate the costs of the test, associated consumables and labour required to perform the test both in the laboratory and the physician's office. The costs of the T-SPOT.TB test were subsequently explored in a sensitivity analysis.

\section{RESULTS}

Figure 1 shows how the costs, combined with the actual clinical data, allow the full costs of each clinical pathway to be calculated. The total cost of the TST only pathway (model 1) is CHF1,078,790 (€695,820), the initial cost of screening 1,000 contacts with the TST is CHF35,000 $(€ 22,575)$ and the costs of subsequently treating the 723 TST-positive individuals for LTBI is CHF1,043,790 (€673,245). The overall cost of using TSPOT.TB only (model 2) is lower at CHF600,210 (€387,135) because, although the initial costs of screening are higher, fewer people receive treatment. As treatment costs are substantially higher than the costs of screening by either test, this results in an overall cost saving. Model 3 is marginally cheaper than model 2, costing CHF531,105 (€342,563) for 1,000 patients.

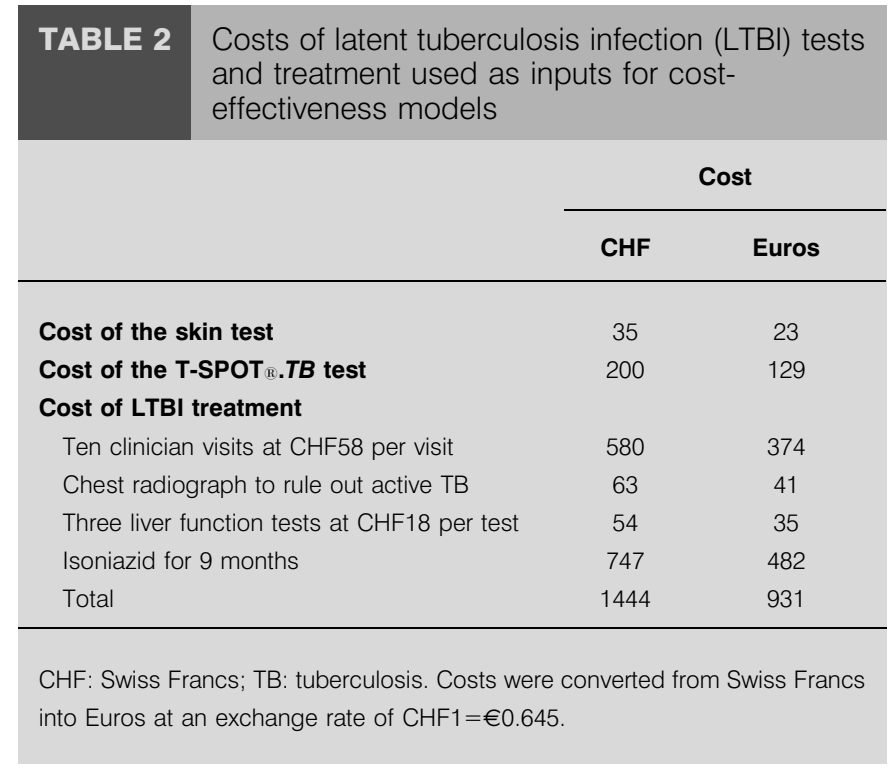

These results show that the costs of an LTBI screening and treatment programme targeted solely at high-risk groups currently costs CHF1,079 (€696) per patient (table 3). Switching solely to the T-SPOT.TB test in place of the TST would reduce costs by $44 \%$ and using both tests together according to the clinical pathway in model 3 would reduce costs by $49 \%$.

A sensitivity analysis of the cost of the T-SPOT.TB test was also performed (table 4); this calculated the overall cost of the TSPOT.TB test at which models 2 and 3, respectively, would become cost neutral to the current testing paradigm (model 1). The results show that if T-SPOT.TB was used as a direct replacement for the TST it would be cost neutral at an overall cost of CHF679 (€438). If T-SPOT.TB was used as a subsequent test after an initial TST, this strategy would be cost neutral at an overall cost for T-SPOT.TB of CHF958 (€618). The cost of TSPOT.TB at which using it alone would be the cheapest strategy was calculated; model 2 would become dominant if the price of T.SPOT.TB was $\leqslant$ CHF131 (€84).

As the induration size for the TSTs was recorded, modelling of the effect of applying different TST cut-offs on the resulting cost of the three screening strategies was also possible. The results are shown in figure 2 . Results with TST cut-off values of 5,6 and $15 \mathrm{~mm}$ were calculated from the clinical data, as these cut-offs correspond to those used in other European countries. The costs of model 2 (T-SPOT.TB only) clearly do not change with TST cut-off, as this screening strategy does not involve the TST. However, as increasing TST cut-off decreases the number of people deemed to be infected, the costs of both models 1 and 3 decrease with increase in the cut-off size from 10 to $15 \mathrm{~mm}$ (to 59 and $70 \%$ over the $10-\mathrm{mm}$ totals, respectively). Decreasing the TST cut-off value from 10 to $5 \mathrm{~mm}$ increases the costs of models 1 and 3, although this effect is relatively small (10 and $7 \%$ increase, respectively). Nonetheless, at all TST cut-off values modelled, the relative costs of each screening strategy were consistent; with model 3 being the cheapest, followed by model 2 and, lastly, model 1 . As higher TST cut-offs increase the specificity of the TST at the cost of sensitivity, the current authors also calculated the proportion of the cohort who were T-SPOT.TB positive but TST negative, as a surrogate for the risk of TST false-negatives. The risk of TST false-negatives increased from 1.9 to $10.1 \%$ as the TST cut-off increased from 5 to $15 \mathrm{~mm}$ (fig. 2).

\begin{tabular}{lcc}
\hline TABLE 3 & $\begin{array}{l}\text { Overall costs of a latent tuberculosis infection } \\
\text { screening and treatment programme }\end{array}$ \\
& Total costs of each screening strategy \\
\hline Model 1 & Model 2+ $^{\text {* }}$ & Model 3 $^{\text {§ }}$ \\
\hline CHF1078790 & CHF600210 & CHF531105 \\
$€ 695820$ & $€ 387135$ & $€ 342563$ \\
\hline
\end{tabular}

Results expressed for a cohort of 1,000 patients. TST: tuberculin skin test; CHF Swiss Francs. ${ }^{*}:$ TST cut-off is $10 \mathrm{~mm}$. ${ }^{\text {: }}$ TST only; ${ }^{+}$: T-SPOT ${ }_{в}$.TB only; ${ }^{\text {s: }}$ TST followed by T-SPOT.TB. 


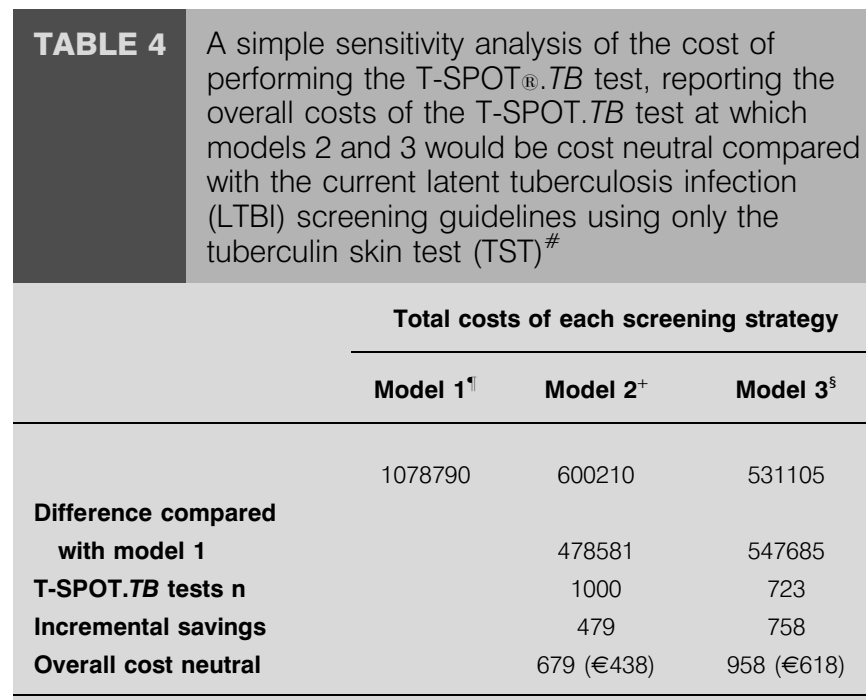

Data are presented in Swiss Francs, unless otherwise indicated. ${ }^{\#}$ : TST cut-off $10 \mathrm{~mm} .{ }^{\uparrow}:$ TST only. ${ }^{+}:$T-SPOT.TB only; ${ }^{\text {s. }}$ TST followed by T-SPOT.TB.

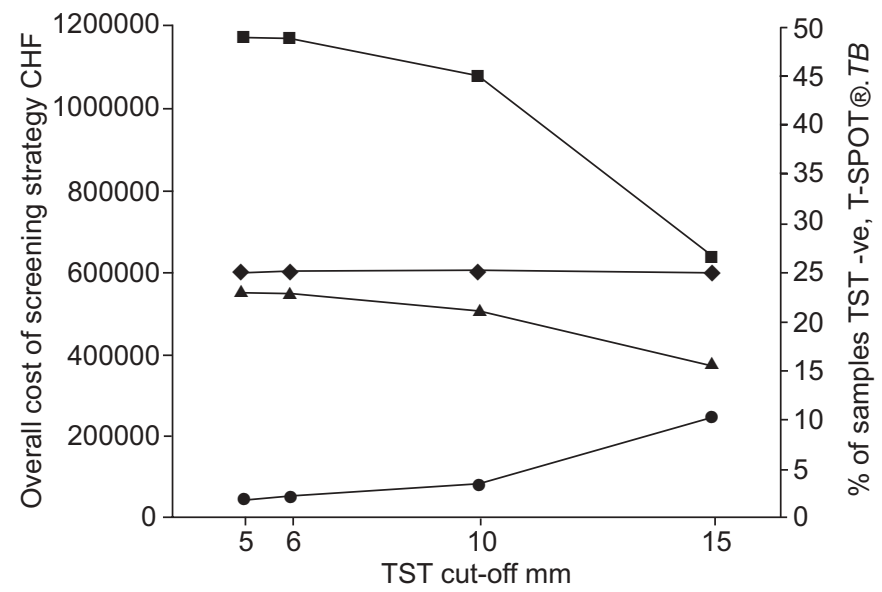

FIGURE 2. The variation of the overall costs of the three screening strategies, and the risk of false-negative tuberculin skin test (TST) results (approximated as those samples that were TST -ve but T-SPOT®. TB +ve) with TST cut-off. $\mathbf{a}$ : Model 1 (TST only); : model 2 (T-SPOT SPOT

\section{DISCUSSION}

Previous cost-effectiveness methodologies for LTBI screening and treatment have used iterative Markov processes to examine the overall costs to the healthcare system over periods of 15-20 yrs [22-28], and have included the costs of TB reactivation and drug side-effects, with or without including the costs of loss of quality-of-life and the costs of wider TB transmission. The present authors chose instead to focus only on direct costs over a much shorter time period, for a number of reasons.

First, the present authors wanted the model to use only actual clinical data, rather than epidemiological assumptions derived from the literature that may or may not accurately reflect the local situation [29]. By taking this approach, it was possible to use the clinical results of the present authors' 2-yr long comparison of both tests side-by-side. The results therefore reflect the reality of the TB screening and treatment programme in a low-incidence region, such as Lausanne, Switzerland.

Secondly, many individual hospitals and public health services, when faced with increasing pressure on healthcare costs and a fixed annual budget to provide TB control, will quite legitimately be examining how the introduction of new technology will impact their annual budget, rather than the overall costs of TB to wider society over a 20-yr timeframe. The restriction of the present model to a simple clinical pathway enables healthcare payers to quickly calculate the actual direct costs of implementing the T-SPOT.TB test and how it will affect their yearly budgets for TB control.

Lastly, the simplicity of the model construction allows the model to be quickly and easily applied to different geographical locations and screening situations, based on actual clinical data that can be easily obtained from pilot studies.

The results of the present study demonstrate that the cheapest clinical pathway in all situations modelled was model 3; using an initial TST followed by T-SPOT.TB only in TST-positive individuals. This finding is consistent with the findings and recommendations presented in the UK NICE Guidelines [21]. It is interesting that this overall conclusion is not affected by the TST cut-off used; and makes the findings from this study potentially applicable to different countries where different TST cut-off values are used. Clearly the potential difficulty of implementing model 3 into clinical practice is that it does not take into account people who may be TST negative, but TSPOT.TB positive. These people may be at risk of TB reactivation, but would not receive treatment. In the cohort examined in Lausanne, this was not a significant factor as there were only nine such individuals in the 267 contacts, representing $3.3 \%$ of all results.

However, if populations are considered where the TST suffers from considerable anergy (for example, young children, and HIV and other immunosuppressed patients), where it is likely that T-SPOT.TB will identify truly infected patients missed by the TST [17, 30-32], then model 3 may no longer be appropriate. Clearly, this argument would also apply to other populations where there is a hidden risk of immunosuppression, such as immigrants with a high probability of undeclared HIV infection. In addition, the present study's results show that in situations where higher TST cut-offs are used, the risk of false-negative TST results can be significant; $10.1 \%$ of the present study cohort would have been considered infected by T-SPOT.TB but not by the TST, if a cut-off value of $15 \mathrm{~mm}$ had been used. To truly understand the cost saving that would result from using T-SPOT.TB in these populations, either on its own (model 2) or according to another clinical algorithm, would require a Markov-type analysis that could compute the cost-savings from the cases of TB averted by early identification and treatment of people at high risk of reactivation. This was outside the scope of the present study, but this is clearly an important area for future research. 
Notwithstanding this future analysis, as the difference in cost was relatively small $(11.5 \%)$ between models 2 and 3 , the clarity of a simplified testing algorithm, the convenience of fewer return visits and the clinical benefit of fewer potential false-negative results afforded by using only T-SPOT.TB may nonetheless make model 2 the preferred strategy over time. Similarly, if the cost of the test could be reduced to $<$ CHF131, then model 2 also becomes the cheapest option, in addition to these other benefits.

To the best of the present authors' knowledge, this is the first analysis of the costs of using a new blood test for tuberculosis infection. Although the detailed costs may change, the present authors believe that the overall results are likely to be applicable to any developed world setting where there is a high proportion of positive tuberculin skin test results, many of whom are due to prior Bacillus Calmette-Guerin vaccination in adults [33] and children [34], and where the main cost of the tuberculosis control programme is thus due to preventive treatment of individuals identified to be infected by the tuberculin skin test. The present results show that using T$\mathrm{SPOT}_{\circledR} . T B$ is likely to bring about substantial direct cost savings in tuberculosis control programmes.

\section{ACKNOWLEDGEMENTS}

The authors would like to thank the Laboratoire BBR Lausanne (director A. Zellweger) and the local staff for running the TSPOT®.TB tests.

\section{REFERENCES}

1 Broekmans JF, Migliori GB, Rieder HL, et al. European framework for tuberculosis control and elimination in countries with a low incidence. Recommendations of the World Health Organization (WHO), International Union Against Tuberculosis and Lung Disease (IUATLD) and Royal Netherlands Tuberculosis Association (KNCV) Working Group. Eur Respir J 2002; 19: 765-775.

2 Lalvani A, Pathan A, McShane $\mathrm{H}$, et al. Rapid detection of mycobacterium tuberculosis infection by enumeration of antigen-specific T cells. Am J Respir Crit Care Med 2001; 163: 824-828.

3 Barnes PF. Diagnosing latent tuberculosis infection: the 100-year upgrade. Am J Respir Crit Care Med 2001; 163: 807-808.

4 Lalvani A. Spotting latent infection: the path to better tuberculosis control. Thorax 2003; 58: 916-918.

5 Behr MA, Wilson MA, Gill WP, et al. Comparative genomics of BCG vaccines by whole-genome DNA microarray. Science 1999; 284: 1520-1523.

6 Andersen P, Munk ME, Pollock JM, Doherty TM. Specific immune-based diagnosis of tuberculosis. Lancet 2000; 356 1099-1104.

7 Lalvani A, Pathan A, Durkan H, et al. Enhanced contact tracing and spatial tracking of Mycobacterium tuberculosis infection by enumeration of antigen-specific T cells. Lancet 2001; 357: 2017-2021.

8 Chapman A, Munkanta M, Wilkinson K, et al. Rapid detection of active and latent tuberculosis infection in HIVpositive individuals by enumeration of Mycobacterium tuberculosis-specific T cells. AIDS 2002; 16: 2285-2293.
9 Ewer K, Deeks J, Alvarez L, et al. Comparison of T-cellbased assay with tuberculin skin test for diagnosis of Mycobacterium tuberculosis infection in a school tuberculosis outbreak. Lancet 2003; 361: 1168-1173.

10 Richeldi L, Ewer K, Bergamini B, et al. T cell-based tracking of multidrug resistant tuberculosis infection after brief exposure. Am J Respir Crit Care Med 2004; 170: 288-294.

11 Richeldi L, Ewer K, Losi M, et al. Early diagnosis of subclinical multidrug-resistant tuberculosis. Annals Internal Med 2004; 140: 709-713.

12 Liebeschuetz S, Bamber S, Ewer K, Deeks J, Pathan A, Lalvani A. Diagnosis of tuberculosis in South African children with a T-cell-based assay: a prospective cohort study. Lancet 2004; 364: 2196-2203.

13 Zellweger J-P, Zellweger A, Ansermet S, de Senarclens B, Wrighton-Smith P. New T cell based test correlated better with tuberculosis exposure than tuberculin skin test. Int $J$ Tuberc Lung Dis 2005; 9: 1242-1247.

14 Meier T, Eulenbruch H, Wrighton-Smith P, Enders G, Regnath T. Evaluation of a new commercial enzyme-linked immunospot assay (T-SPOT.TB) for the diagnosis of tuberculosis in routine clinical practice. Eur J Clin Microbiol Infect Dis 2005; 24: 529-536.

15 Shams H, Weis S, Klucar P, et al. Enzyme-linked immunospot and tuberculin skin testing to detect latent tuberculosis infection. Am J Respir Crit Care Med 2005; 172: 1161-1168.

16 Soysal A, Millington K, Bakir M, et al. Effect of BCG vaccination on risk of Mycobacterium tuberculosis infection in children with household tuberculosis contact: a prospective community-based study. Lancet 2005; 366: 1443-1451.

17 Dheda K, Lalvani A, Miller R, et al. Performance of a T-cellbased diagnostic test for tuberculosis infection in HIVinfected individuals is independent of CD4 cell count. AIDS 2005; 19: 2038-2041.

18 Mori T, Sakatani M, Yamagishi F, et al. Specific detection of tuberculosis infection: an interferon-gamma-based assay using new antigens. Am J Respir Crit Care Med 2004; 170: 59-64.

19 Brock I, Weldingh K, Lillebaek T, Follmann F, Andersen P. Comparison of tuberculin skin test and new specific blood test in tuberculosis contacts. Am J Respir Crit Care Med 2004; 170: 65-69.

20 Ligue pulmonaire suisse et Office fédéral de la santé publique. Manuel de la tuberculose. Berne, Ligue Pulmonaire Suisse, 2003; pp. 1-37.

21 National Institute for Clinical Excellence (NICE). Tuberculosis. National clinical guideline for diagnosis, management, prevention, and control. Wetherby, UK, National Institute for Clinical Excellence, 2006.

22 Dasgupta K, Schwartzman K, Marchand R, Tennenbaum T, Brassard P, Menzies D. Comparison of cost-effectiveness of tuberculosis screening of close contacts and foreign-born populations. Am J Respir Crit Care Med 2000; 162: 2079-2086.

23 Diel R, Nienhaus A, Schaberg T. Cost-effectiveness of isoniazid chemoprevention in close contacts. Eur Respir J 2005; 26: 465-473.

24 Salpeter S, Salpeter E. Screening and treatment of latent tuberculosis among healthcare workers at low, moderate 
and high risk for tuberculosis exposure: a cost-effectiveness analysis. Infect Control Hosp Epidemiol 2004; 25: 1056-1061.

25 Schwartzman K, Menzies D. Tuberculosis screening of immigrants to low-prevalence countries. A cost-effectiveness analysis. Am J Respir Crit Care Med 2000; 161: 780-789.

26 Salpeter S, Sanders G, Salpeter E, Owens D. Monitored isoniazid prophylaxis for low-risk tuberculin reactors older than 35 years of age. A risk-benefit and cost-effectiveness analysis. Ann Intern Med 1997; 127: 1051-1061.

27 Rose D. Short-course prophylaxis against tuberculosis in HIV-infected persons. A decision and cost-effectiveness analysis. Ann Intern Med 1998; 129: 779-786.

28 Schechter C, Rose D, Fahs M, Silver A. Tuberculin screening: cost-effectiveness analysis of various testing schedules. Am J Prev Med 1990; 6: 167-175.

29 Walker D. Economic analysis of tuberculosis diagnostic tests in disease control: how can it be modelled and what additional information is needed? Int J Tuberc Lung Dis 2001; 5: 1099-1108.
30 Huebner R, Schein M. The tuberculin skin test. Clin Infect Dis 1993; 17: 968-975.

31 Anastos K, Kalish LA, Palacio H, et al. Prevalence of and risk factors for tuberculin positivity and skin test anergy in HIV-1-infected and uninfected at-risk women. Women's Interagency HIV Study (WIHS). J Acquir Immune Defic Syndr 1999; 21: 141-147.

32 Johnson JL, Nyole S, Okwera A, et al. Instability of tuberculin and Candida skin test reactivity in HIV-infected Ugandans. The Uganda-Case Western Reserve University Research Collaboration. Am J Respir Crit Care Med 1998; 158: 1790-1796.

33 Tissot F, Zanetti G, Francioli P, Zellweger JP, Zysset F. Influence of bacille Calmette-Guerin vaccination on size of tuberculin skin test reaction: to what size? Clin Infect Dis 2005; 40: 211-217.

34 Collet E, Krahenbuhl JD, Gehri M, Bissery A, Zellweger JP. Risk factors for positive tuberculin skin tests among migrant and resident children in Lausanne, Switzerland. Swiss Med Wkly 2005; 135: 703-709. 